\title{
Research on Academic Burden from International Perspective
}

\author{
SongzhuMei ${ }^{1}$, Zhengxin Zhu $^{2}$,PingLeng ${ }^{3 *}$ \\ ${ }^{1}$ Educational College, Huaibei Normal University, Huaibei Anhui, China \\ ${ }^{2}$ School of Language and Culture, Shanghai Normal University Tianhua College, Shanghai, China \\ ${ }^{3}$ College of Mathematic and Science, Huaibei Normal University, Huaibei Anhui, China \\ *Corresponding Author.
}

\begin{abstract}
Academic burnout of basic education is widespread in varying degrees in the global scope, and such situation is particularly serious in Chinese primary and secondary schools. International research has rarely mentioned the concept of academic burden, which is mainly described in other similar concepts, such as academic burnout, academic stress, academic depression and academic anxiety. In addition, studies in other countries have paid more attention to the negative influence of academic load on students' body and mind. Based on international perspective, the paper clarifies the somatic and psychological symptoms, behavior and attitude symptoms and symptoms of academic burnout caused by heavy academic burden, analyzes the influence factors of academic burden, and compares the function and applied scope of inventories and scales for academic burden. Findings enlighten us to focus on the teaching situations and students' nature, increase empirical research and quantitative research of academic burden, put forward evidence-based, operational and pragmatic strategy of alleviating academic burden.
\end{abstract}

Keywords: Lassitude, stress, intervention, scale, academic burden

\section{Introduction}

Since the foundation of new China, academic burden in basic education has always been criticized by the general public and is regarded as one of the difficult points of educational reform. Current strange phenomenon that academic burden in Chinese primary and secondary schools is overloaded is caused by combined factors, such as the examination system only focusing on test scores, scarce high quality educational resources, knowledge-based instructing approaches and fiercely competitive employment environment. Although government has taken actions of "alleviating academic burden on students" (burden alleviation), the implementation effect is not very satisfying, even getting counterproductive results. Overloaded academic burden tortures students, parents and teachers and has become the stubborn problem of China's education. Based on the international view and referencing experience from other countries, it would be a profitable attempt to explore sound strategies in burden alleviation from investigating the influence factors, production mechanism, and intervening measures on academic burden.

\section{Distinction of Concepts of Academic Burden}

In order to focusing the theme, it is very necessary to retrospect the lexical origins and intensive expansion of related concepts. Schoolwork is also known as assignments, while academic missions refer to assignments of knowledge, academic scholarism and learning. Burden in Chinese means undertaking or bearing, when used as verb, while refers to individuals' work, or pressure and responsibilities burdened physically and mentally as noun. The concept of "academic burden" was firstly introduced from the paper Actively Trying to Relieve the Students of the Heavy Academic Burden, published on People's Education in 1954, which was also the origin of the concept "burden alleviation". In other words, the original concept of "burden alleviation" in Chinese context meant alleviating homework load, which was as similar as the concepts in South Korea, Japan and Singapore. As time goes on, the connotation of this concept has been changed. Chen Shuangye mentioned that the academic burden in China has transcended the conceptual category in international research area, which is the integrated effects of learning duration, quantity, energy, emotion input, and pressure feelings. ${ }^{[1]}$ Song Naiqing pointed that academic burden is the pressure experience and the time and energy spent in, which triggered by test evaluations and assignments, when students are trying to adapt to current learning environment. ${ }^{[2]}$ It follows that "burden alleviation" in China is no longer a method only relieving the burden of schoolwork, but the one endowed much more connotations and expectations. Similar concepts also include schoolwork burden, learning burden, students'

ISSN: 0010-8189

C CONVERTER 2021

www.converter-magazine.info 
burden. In a broad sense, academic burden refers to all of the learning assignments undertaken from school, society, family and requirements made by students themselves. ${ }^{[3]}$ In a narrow sense, academic burden is the total amount of assignments arranged by schools. In this condition, learning tasks and the whole assignments are not only stipulated in quantity but also in quality, as well as the physical and mental reactions caused by it. "Learning burden" or "students' load" are two more colloquial concepts. In specific contexts, these concepts could be used to represent "academic burden" or "schoolwork burden", and will become blurring without the contexts. Compared with the above concepts, it is not very difficult to find that the "burden alleviation" mentioned repeatedly in China's basic education is actually alleviating generalized "academic burden", that is to say, alleviating schoolwork burden in campus and relieving learning load from afterschool tutoring institutions and students themselves. The ultimate aim is not only to reach the decrease of the "quantity" of dominant burden, but also the "quality" of recessive burdens in psychology, physiology and emotion.

Academic circle in China has made longstanding research on academic burden. Although the achievements in theoretical suppositions and mechanism analysis are fruitful, many weaknesses have also been exposed: Due to the inconsistent concept names and connotation demarcation, research points are not coincident; Out of the difference in research angles and interest subjects, the situation of mutual shuffling and no consensus is formed; Rational thoughts are the mainstream research thoughts, while less empirical studies and international comparative studies are presented. Till now, the problems of academic burden are still the hotspot and difficulty of China's basic education reform and research. However to define, such situations basically contain pressure, tasks, reactions and other key words. At the same time, some common core expressions are existed: firstly, integrating objective learning gross with subjective mental feelings; secondly, serious unbalanced results caused by learning content, time and patterns; thirdly, complicated confronts between multiple subjects and logics; forth, possessing properties, such as relativity, variability, durability, and etc.

In the international research field, "academic burden" and "schoolwork load" are seldom mentioned, the reasons are as follows: Firstly, academic burdens in other countries are not as serious as China. However, the situations in South Korea, Japan and Singapore, and other Asian countries are another matter. Secondly, scholars in other countries have different research angles. They don't intend to struggle on "whether the burden should be relieved" and "administrative responsibilities". Rather, they put their attention on exploring students' physical and mental problems caused by academic burden, as well as the generation mechanism and external intervention of those problems. Based on such views, international research pays more attention on the negative effects, such as physical dysfunction, exhausted emotional fluctuation, psychological depression and anxiety, negative and passive attitudes, garbled and disrupted personalities, decline of efficiency performance, which caused by schoolwork and learning process. Thus, related conceptual system has been developed. In 1980s, the United States and other western countries created concepts of learning burnout, academic burnout, academic pressure, academic anxiety, etc., to describe the mental and physical damages on students caused by long term and high expected schoolwork content and learning requirements. Ayala M. Pines firstly introduced the concept of "Studying Burnout" in the book Burnout: From Tedium to Growth. Scott T. Meier had defined "learning burnout" as the phenomenon of energy exhausted, gradual decline of enthusiasm in learning activities, indifferent and isolated learning attitude and taking a negative attitude on schoolwork, out of long term academic pressure and learning load. ${ }^{[4]}$ Yvonne Gold defined academic burnout as the exhausted feeling caused by exorbitant academic demands, which would let students trap in an academic inefficiency because of the isolated and sarcastic attitudes. ${ }^{[5]}$ James P. Kohn (1986) believed that academic stress was a kind of stress reaction, physically and psychologically, caused by schoolwork related stressor. ${ }^{[6]}$ Academic anxiety is a series of negative results formed when individuals facing specific learning situation, such as discomfort, alienation on learning, poor academic performance or related issuesAbove concepts are widely used in international research to describe the multiple damages on students caused by academic load. However, some problems exist in these concepts. For example, such concepts are lack of accuracy. The concepts of pressure, anxiety, depression, load and etc. are hard to distinguish. Directivity of concepts is hard to clarify from stimulus or pressure experience. ${ }^{[7]}$ Such situation will lead to the challenging towards concepts. Unscientific pop-psychology bias and lack of empirical evidence will threat the existence of concepts themselves. After comparing international and domestic concepts, we could find that the former tend to describe and measure various symptoms of academic burden, while the latter keens on speculation and generalization on the reasons of academic burden and burden alleviation strategies. In addition, the former is specific and operable, while the latter is more macroscopic and difficult to execute. The reasons are not only caused by the difference of codes of language, but also the difference in learning, but the essence and directivity are consistent, that is when exploring the negative influence of academic burden on students' body and mind in various aspects, the two aspects could complement each other. Therefore, when scholars run studies on academic burden from the perspective of international range, they shouldn't simply copy the old-fashioned research approaches, but discuss from the angles of academic burden symptoms, influence factors and intervening measures.

ISSN: 0010-8189

(C) CONVERTER 2021

www.converter-magazine.info 


\section{Symptoms of Academic Burden}

\subsection{Physical symptoms}

Many studies have shown that dominant results of academic overburden are causing a variety of diseases. David F. Gillespiea (1982) found that the correlation coefficient between academic burden and body health was between- 0.16 to -0.44 based on quantitative analysis. ${ }^{[8]}$ Teija Honkonena (2006) believed that academic burden would erode human health chronically, which may result in skeletal muscle diseases, cardiovascular diseases, idiosyncrasy, anaphylactic reactions, and anaphylactic reactions. ${ }^{[9]}$ However, Sophia Kahill stated that the evidence of attributing generalized body diseases to academic burden was obviously insufficient. Only the diseases, such as insomnia, nightmare, headache, stomachache, intestinal flora, fatigued weary, and physical power exhausted, may have stronger connection with academic burden. ${ }^{[10]}$ In addition, onerous and tedious academic burden deprive students' exercise and breaks. This may lead to the gradual decline of physical agility, dramatic descending of vision and rapid changes of weight, which have been reflected in previous news reports..

\subsection{Psychological symptoms}

Academic overburden will not only trigger body diseases, but also influence mental health, resulting in depression, anxiety, pressure and other psychological problems.

\subsubsection{Depression}

When students suffer from long-term academic overburden, their mind and emotion will be influenced directly, causing frequent, continuous, and furious psychic reaction and further resulting in depression. Common symptoms of depression include crying, annoying, irritability, secret sadness, pessimism, self-accusation and compunction, self-hatred, self-accusing, suicide ideas, losing interest, hesitation, physical exhaustion, lack of vitality, somnipathy, appetite change, sense of failure, sense of punishment, sense of worthless, being unable to concentrate. ${ }^{[11]}$ Max Hamilton described depression as emotional depression, sense of guilty, bluntness, annoying, anxiety, body symptoms, genital symptoms, hypochondriasis, decline of vision and weight, schizobulia, paranoia, and etc. ${ }^{[12]}$ There are many reasons which may trigger depression and could be classified into two main groups: firstly, biological factors, such as brain chemistry, hormone, Sub-threshold Stimulation, genetics, imbalance of cranial nerve and medicinal influence; secondly, socil psychological factors, like environmental stress and important life events. When students can no longer bear the academic burden, or forced by huge external pressure, their bioinformatics will lead to stress reaction. With the accompanying of furious psychological effects, depressive symptoms will occur.

\subsubsection{Anxiety.}

Anxiety is another kind of psychological symptom triggered by academic burden. It is differ from depression, but sharing some similarities. Sigmund Freud (1936) declared that anxiety was an emotional state of perception, which was expressed by fear, worriment, tension, disturbance, even accompanying physiological arouse; from the perspective of Darwin's theory, anxiety is a kind of motivating behavior, which can help ideal adaptation and responding to external threats. When facing high load academic burden, if students don't possess the strategies and abilities to deal with such burden, anxiety will be triggered. Anxiety is usually expressed in two forms: One is physiological anxiety, shown through nervous and restless, and not know what to do, and the serious conditions could be sweating, tachycardia, body trembling, breathing quickly and other physiological phenomenon; another is cognitive anxiety, which is a kind of cognitive bias caused by worried about bad evaluations or punishment of outside world, even some extreme ideas of regarding it as a disgrace. ${ }^{[13]}$ Academic burden can trigger both of the anxieties. For students who have high self-requirements, bear high expectations from outside world, and have poor capacity of psychological enduring and adaptability, they are easier to be disturbed by such problems.

\subsubsection{Pressure}

As a kind of external stimulation, which interacts with students' study and life, academic burden will cause feeling experience and emotional conditions accompanying with anxiety and worriment, and further form visible or invisible pressure. Hans Selye (1936) thought stress was a kind of non-reifiable body reaction under any change demand. It is usually use to refer to the negative influence when individuals face unpleasant situations or objects. Pressure is itself, its causes, and its resultsLong term academic burden will make students always trap in nervous and anxious conditions and be indecisive and hesitated because of poor performance. If such situation goes, chronic mental fatigue and pressure will be formed. Not only physical disease will occur, like palpitation, stethalgia, myocardial infarction, headache, night sweat, anabrosis, but also psychic disarrays and behavior ISSN: 0010-8189 
disorders, such as emotional disorders, anxiety disorders, suicidal tendency, substance use disorder, eating disorders, single pole depression, bipolar disorders, schizophrenia, and etc. ${ }^{[14]}$ Pressure symptoms are the typical results of academic burden. It exists all the time, while different individuals have different feeling degrees.

\subsection{Symptoms of behaviors and attitudes}

Academic burden is filled throughout students' life. Not only their mind and body health will be influenced, but also their behaviors, attitudes and academic performance. ${ }^{[15]}$ In the life aspect, their living habits will be changed, such as staying up late, sleepiness, disordered daily routine, anticlimax, inadvertence, indecision. What's worse, extreme behavior may occur, such as alcoholic abuse, smoking, apastia, overeating, drug dependence and abuse. In the academic aspect, students may become being late and leaving early, absence, laziness, inattention, performance decrease. In the aspect of interpersonal communication, self-closing, isolation, indifference, brutality, inhumanity, shouting, and damaging goods will occur, even the tendency of self-harm and suicide. In the perspective of value, self-denial, self-contradiction, self-depreciation will also be formed, even pessimism, misanthropy, desperation, self-defense, insensitivity, dehumanization, cynicism, escaping from groups. All such behaviors and attitudes may be not existed in one person at the same time, but as the group tortured by academic burden, students are frequently suffered from the above problems, even an individual possessing multiple symptoms which seem contradictory at the same time.

\subsection{Academic burnout}

Another negative effect of academic burden is the academic burnout. It refers to the emotion exhausted, cynicism, and declining of expressive force, caused by chronic academic pressure and schoolwork load, which deviate from students' learning needs. ${ }^{[16]}$ Academic burnout starts from the framework of occupational burnout. It is a concept which believes motion exhausted, cynicism, and declining of expressive force are immanent problems existing among students. This will lead to burnout symptoms existence at the beginning of students' career and will affect the future occupation. At present, college students are mainly the objects of international research on academic burnout, while less has been focused on students in primary and secondary school. Study showed that the groups with relatively high self-esteem and self-identification are hard to be suffered from academic burnout. ${ }^{[17]}$ Academic burnout will increase accompanying with students' age. ${ }^{[18]}$ Students' emotional exhaustion is mainly caused by the separation between current academic requirements and expected effectiveness and efficiency, which leads to the further cynicism and low efficiency towards study ${ }^{[19]}$ Because of the individual difference and various social support, symptoms of body, mental, behaviors and attitudes caused by academic burden may be not inevitable or obvious enough. However, the burnout caused by academic burden is almost universally existed. Therefore, researchers in other countries generally focus on academic burnout, and try to find out scientific tools to measure and evaluate the burnout, as well as the evidence of external intervention influence on it.

\section{Influence Factors on Academic Burden}

In China's academic circle, academic overburden is always attributed to many factors, including official based culture origin as "Officialdom is the natural outlet for good scholars.", utilitarianism under the system, peer effects of prisoner's dilemma game, repeated deduction of high vital examination, score-based exam-oriented education pattern, evaluation mechanism partially pursuing enrolment rate, traditional pedagogy on weighing knowledge more than techniques, improperly designed curriculum and teaching materials, unrealistic parents' expectation, shortage and maldistribution of high-quality educational resources, outdated teaching methodology and ideas, employment system only relying on certificates, and etc. It is not difficult to find that such attributive pattern mainly bases on the views of culture, system and surroundings, macroscopically and indistinctly, which are unique in China. At the international level, although some researchers have discussed the reasons why academic burden caused from the above angles, more focus on school field, that is exploring academic burden caused by contradictions among curriculum, teaching, study and evaluation.

International researchers all agree that examination is the most important inducement of academic burden, especially the ones with high importance and standardization, which bring pressure far beyond students' acceptance and controllable range. ${ }^{[20]}$ Exam-based teaching limits students' identity and objective status and students will be over anxious because of the fail in the tests. ${ }^{[21]}$ Students' emotional happiness will dramatically decrease under the effect of exams, so that academic pressure is born and academic burden formed.

In China, except exams, academic pressure comes from four main aspects: Firstly, the social pressure: The rooted social values influenced by traditional Confucian ideology has gone beyond the pure academic field, which makes ISSN: 0010-8189

(C) CONVERTER 2021

www.converter-magazine.info 
learning targets pursued under utilitarianism becoming the social collective unconsciousness. Secondly, the expectation pressure: traditional core virtue, Filial Piety, makes students obey parents' will to comply with parents' high expectation and familial responsibility framework choice, just out of their own self-respects. At the same time, teachers' expectations and students own achievement expectations will also take huge driving functions. ${ }^{[22]}$ Thirdly, peers pressure: Peer competition, encouragement and tease will pass group norms and behavior imitation, which constructs pressing phenomenon. ${ }^{[23]}$ Fourthly, educational environment pressure: Traditional teaching methods, strained relationship between teachers and students, heavy schoolwork, frequent high-vital exams, and uncomfortable school environment will lead to academic pressure ${ }^{[24]}$ Research showed that academic pressure is related to gender, race and socioeconomic status. Generally, female students' pressure is higher than male ones; Asian students have higher pressure than peers in other countries; pressure increase accompanying with grades, and students in graduating class have more stress than students in other grades; when the demands increases, while self-control and social support decrease, pressure will rise accordingly. ${ }^{[25}$ Therefore, academic pressure is both the important origin of academic burden and the important reflection of academic burden.

\section{Measurement of Academic Burden}

Academic overburden has very wide negative effects on individuals. Body, mind, emotion, cognition, communication and etc. will be damaged or impeded by it. In order to efficiently measure the influential aspects and levels, a series of operable scales, questionnaires, diagnostic manuals are introduced. Structured Clinical Interview for DSM-III-R could be used to make overall assessments on students various disorders, including mental disorders, emotional disorders, substance use disorders, anxiety disorders, physical impairments, eating disorders, adjustment disorders and personality disorders. ${ }^{[26}$ Somatic Complaints and Burnout and Children's Somatization Inventory could be used to analyze the influence of academic burden on students' body. Depression Anxiety Stress Scales could be used to assess depression, anxiety and pressure caused by academic burden at the same time, which makes up the shortage in distinguishing depression and anxiety in traditional approaches. The detailed version contains 42 items while abridged one contains 21 items. The latter seems better than the former. ${ }^{[27]}$

Educational and psychological circle in the United States has also introduced some special scales to measure depression, anxiety and pressure. Authoritative and widely used scales contain Hamilton Rating Scales for Depression, Beck Depression Inventory, Beck Depression Inventory-II, Cognitive Self-Statements in Depression, Children's Depression Inventory and Peer Nomination Inventory of Depression. These scales are varying in item setting and structural composition. They could serve as mutual complementation to roundly measure depressive symptoms caused brought by academic burden. As for measurement on anxiety, Anxiety Status Inventory can be used to provide anxiety diagnostic criteria. Revised Children's Manifest Anxiety Scale is used to assess dominant anxious symptoms caused by academic burden. Beck Anxiety Inventory can separate isostructural and isomerous anxious groups from other psychological groups. ${ }^{[28]}$ Multidimensional Anxiety Scale for Children measures from four-dimensions, including body condition, social anxiety, isolation anxiety, and damage avoidance. ${ }^{[29]}$ Test Anxiety Inventory for Children and Adolescents can be used to measure specific pressure formed in exams. Pressure is both the reasons and results of academic burden. This has become a consensus. Tools used to measure pressure are various, too. Academic Stress Questionnaire, Academic Stress Scale, Student Stress Inventory, Academic Stress Questionnaires can be applied to test students' overall pressure condition. School Stressors Inventory for Adolescents and High School Stressors Scaleare used to measure the origins of academic pressure. Student-Life Stress Inventory and Stress in Children Questionnaire can indicate subjects' reactions under pressure. Survey of Academic Stress and Lakaev Academic Stress Response Scalecan also be used to measure pressure related social factors. In 2011, Educational Stress Scale for Adolescents was introduced. It divides academic pressure into five aspects: learning pressure, schoolwork pressure, worrying about scores, pressure of self-expectation, and learning depression, which relatively measure the pressure related with academic burden in a comprehensive angle. Above scales and questionnaires aim at different student groups. Therefore, they should be chosen and revised before use.

In addition, educational research field in the United States introduced some integrated scales. Quality of Learning Experience is one of those scales, which contains eight parts, namely resources, contents, flexibility of learning, formal and informal interaction between teachers and students, students involvement, emotional exhaustion, perceive achievements for students, students' promise, ${ }^{[30]}$ which could be used to systematically measure the relationship between academic burden and correlated variable. Self-Control Schedule, Brief Fear of Negative Evaluation Scale, Hopelessness Scale for Children, Crandell Cognitions Inventory and Multidimensional Scale of Perceived Social Support could be also used as profitable supplement to measure academic burden.

Maslach Burnout Inventory measures occupational burnout from three dimensions: emotional exhaustion, ISSN: 0010-8189

(C) CONVERTER 2021

www.converter-magazine.info 
removing humanization, decline of individual achievements. ${ }^{[31]}$ Based on this, 15 items are set in MBI-SS to use the same dimensions to measure students' learning burnout. This scale had been translated into Chinese and its internal consistency reliability coefficient was ideal. ${ }^{[32]}$ Besides, Oldenburg Burnout Inventory-Studentsis set into two dimensions: Exhaustion and Disengagement, which has solved the problem of assignment of plus or minus directions in MBI-SS. ${ }^{[33]}$ Academic Self-Regulation Questionnaire can also be used to measure primary and secondary school students' academic burnout. This questionnaire contains five dimensions, including external rules, inclusive rules, identifying rules, intrinsic motivation, and lack of motivation, 30 items in total. ${ }^{[34]}$ In those scales, except three elements in the Maslach's scale, gender, socioeconomic status, negative personality characteristics, flexibility of problem solving, learning targets, learning objectives, social support, negative campus phenomenon and other factors are all paid special attention to find their influence on academic burnout.

Currently, when studying academic burnouts, some researchers start to focus on the opposite concept of burnout - Engagement. Engagement is organization members utilize of their own working roles. In role plays, individual use and express himself or herself in body, cognition, and emotion. Disengagement, on the contrary, separates oneself from working roles and shows retreat and defense $\mathrm{n}$ body, cognition, and emotion in role plays. ${ }^{[35]}$ Disengagement is a kind of stress reaction of burnout, as well as the negative performance of avoiding academic burden. As for engagement, Utrecht Work Engagement Scale for Students can be applied to measure students' engagement from three dimensions, namely vitality, dedication and concentration. ${ }^{[36]}$

\section{Intervention on Academic Burden}

Too much academic burden will seriously harm students' healthy growth both in mind and body. To fight against this, many domestic scholars suggest to build fair educational environment, strengthen macro-control and supervision, reform entrance exam system, establish rational evaluation system, change administrative burden alleviation, rebuild authority-responsibility relationship on burden alleviation, develop higher education resources, guide society to regard rationally, optimize curriculum and administration, promote teachers' educational quality, and etc. These suggestions make rational plans for system, environment, and ideas, but the implement of each suggestion is not an easy task. Without required situations of providing effectiveness and operability, they are just like mirage.

International scholars believe if burden is supposed to be alleviated, sufficient social support must be provided to students, including emotional and information support. Emotional support refers to providing information for chaos out of sympathy and other emotional expression and the information support means providing information for individual problem solving activities. ${ }^{[37]}$ If the support on emotion and information is increased, academic pressure and depression should be decreased correspondingly. ${ }^{[38]}$ These supports could be positively perceived by students. The perceivable supports will rapidly affect body healthy problems caused by pressure, ${ }^{[39]}$ and can change people's mental state. In a sense, perceivable supports are personality characteristics of self-stability, rather than real supporting behaviors from other people. Then, shaping ideal personalities is an important approach of burden alleviation. The ideal personalities are extrovert, easy-going, opening, responsible and emotionally stable. It could be a kind of handling mechanism and behavioral pattern, as well as rooting inside the resources, even becoming a kind of resource. It could also be a resource required by individuals or deterrence of abnormal behaviors. ${ }^{[40]}$ What's more, cultivating students acquiring the wise of pressure reducing will effectively decrease the influence of pressure on their cognitive performance. It will help individuals norm the internal affairs of their emotion and cognition and form the behavioral and cognitive skills which will help them successfully achieve their goals. ${ }^{[41]}$ When we can't change the external environment, it would be a wise choice to change ourselves to adapt the surroundings.

Reducing academic pressure is another efficient way to alleviating burden. Four key elements of dealing with pressure are environmental pressure source, cognitive appraisal, physical, mental and behavioral experience in pressure level, solving behaviors or strategies. ${ }^{[42]}$ No matter how the processing mode is set based on either problem or emotion, it can eliminate or decrease pressure, or manage or decrease the influence of threatening cases. In addition, taking part in emergency pressure precaution training programs will also decrease the academic pressure. It is a flexible, individual-based and multifaceted cognitive behavior therapy and its purpose is to help individuals dealing with continuous pressure and provide tools to handle pressure in the future, which is generally divided into three phases, including cognitive preparation, skills gaining and rehearsals, and continuous applying. ${ }^{[43]}$

In addition, releasing academic burnout can also decrease academic burden. Appreciable independent support to students, mental control in school environment and working immerse will decrease academic burden in some degree. ${ }^{[4]}$ Some researchers believe that academic burnout comes from students schoolwork resources, such as motivation, social support, problem solving strategies, and success expectations from themselves, teachers, parents ISSN: 0010-8189

(C) CONVERTER 2021

www.converter-magazine.info 
and peers, are not matching properly. ${ }^{[45]}$ Abundant schoolwork resources and proper success expectations could appropriately decrease the level of academic burnout. Besides, many studies showed that the interactive relationship between students and family, teachers, peers, and parents relationship, parenting styles and whether parents experiencing some kind of burnout will affect students' attitudes and behaviors and further influence academic burnout. Therefore, strengthening students' cognition on academic burnout, providing multi-dimension resources and environmental support, improving psychological adjustment will efficiently resist academic burnout, so that the original intention of relieving academic burden could be reached.

\section{Conclusion}

International research on academic burden may differ from domestic studies on concept connotation, research focus, research methods and etc. Such situation leaves us many thinking approaches: Firstly, it is important to make academic analysis on the origin and influence of academic burden from the angles, such as traditional culture, current system, and educational environment. But if the research separates itself from teaching situation and students themselves, it would be rough and void. Even provocative theoretical calling on, only the practicability of operation is missing. Secondly, without practice test and vertical empirical analysis, even the form of burden alleviation is perfect, which is presented from different research subjects, academic burden objects, the achievements are idealistic working behind closed door or scholars' own wishful thinking at best, because the reality always possess complexity and refractoriness that beyond our imagination. Thirdly, interventional methods on academic burden based on pedagogy, and psychology, and sociology and other multi-dimension fields is a positive attempt in burden alleviation. Governments should avoid blindness in burden alleviation out of administrative purpose. Burden alleviation policies and education decisions should be made after fully consideration based on evidence and educational equality. Persistence and consistency of these policies and decisions should also be guaranteed. Fourthly, applying achievements in international research mechanically into China's current national conditions and system may be not a wise choice. However, its research ideas, research methods and rigorous academic attitudes are worthy of profound introspection and wide reference for China's academic circle.

\section{Acknowledgements}

This research was supported by 2020 Key Project of the Chinese 13th Five-Year Plan for National Education and Science(Grant No. DHA200323).

\section{Reference}

[1]S.Y. Chen,K. Zheng. "From personal obsession to public education issues: understanding the schoolwork burden of primary and secondary school students in the real world," Journal of Global Education Outlook, vol.41, no.2,pp.15-23,2013.

[2]N.Q.Song,X.Yang,D.H.Wang. "A study on the evaluation model of students' homework burden -a case study of students in compulsory education," Journal of Southwest University (Social Sciences Edition),vol.41, no.3,pp.75-81,2015.

[3]J.S.Ma,J.N.Wu. "Why is it so hard for student burden reduction policies to bear fruit? on the nature and mechanism of time distribution of academic burden," Journal of Beijing Normal University (Social Sciences Edition), no.2,pp.5-14,2014.

[4]T.Scott. Meier,R.R. Schmeck. "The burned-out college student: a descriptive profile," Journal of College Student Personnel, vol.26, no.1,pp.63-69,1985.

[5]G.Yvonne,B.Patricia,B.M.William. "The dimensionality of a modified form of the maslach burnout inventory for university students in a teacher-training program," Journal of Educational and Psychological Measurement,vol.49, no.3,pp.549-561,1989.

[6]P.K. James,H.F. Gregory. "An academic stress scale: identification and rated importance of academic stressors,” Journal of Psychological Reports,vol.59, no.2,pp.415-426,1986.

[7]P.David. "Researching academic stress and anxiety in students: some methodological considerations," British Educational Research Journal, vol.33, no.2,pp.207-219,2007.

[8] F.G. David. "Correlates for Active and Passive Types of Burnout. Journal of Social Service Research,vol.4, no.2,pp.1-16,1982.

[9]H.Teija, A.Kirsi, P.Marja, et al.,"The association between burnout and physical illness in the general ISSN: 0010-8189

C CONVERTER 2021 
population-results from the finish health 2000 study," Journal of Psychosomatic Research,vol.61, no.1,pp.59-66,2006.

[10]K.Sophia. "Symptoms of professional burnout: a review of the empirical evidence. Journal of Canadian Psychology,vol.29, no.3,pp.284-297,1988.

[11]J.A.D. David, S.D. Keith, L.A.Jamie. "A psychometric evaluation of the beck depressioninventory-II. Journal of Psychological Assessment, vol.10, no.2,pp.83-89,1998.

[12]H. Max. "A rating scale for depression," Journal of Neurology,Neurosurgery and Psychiatry, vol.23, no.56,pp.56-61,1960.

[13]P.F.Lovibond, S.H.Lovibond. "The structure of negative emotional states: comparison of the depression anxiety stress scales (DASS) with the beck depression and anxiety inventories," Behaviour Research and Therapy, vol.33, no.3,pp.335-343,1995.

[14]J.D. Sun, P.D. Michael, X.Y. Hou. "Academic stress among adolescents in China," Australasian Epidemiologist, vol.19, no.1,pp.9-12,1988.

[15]F. Gary, W. Kathy. "Influences of stress, situation-specific mastery beliefs and satisfaction with social support on well-being and academic performance," Psychological Reports, vol.70, no.1,pp.219-303,1992.

[16]Y.Q. Gan, J.Y. Shang, Y.L. Zhang. "Coping Flexibility and locus of control as predictors of burnout among chinese college students," Social Behavior and Personality: An International Journal,vol.35, no.8,pp.1087-1098,2007.

[17]L. Jayoung, P. Ana, Y.B. Kim, et al. “Academic burnout profiles in korean adolescents.” Stress and Health, vol.26, no.5,pp.404-416,2010.

[18] L. Jayoung, P. Ana, L. Eunkyoung, et al.,"Age-related differences in academic burnout of korean adolescents," Psychology in the Schools, vol.50, no.10,pp.1015-1031,2013.

[19]N. Hyunkyung, S. Hyojung, M.L. Sang. "Developmental process of academic burnout among korean middle school students," Learning and Individual Differences, vol.28, no.1,pp.82-89,2013.

[20]Michael J. Connora.Pupil Stress and Standard Assessment Tasks (SATs): An Update. Emotional \& Behavioural Difficulties, vol.8, no.2,pp.101-107,2003.

[21]H. Kathy, C. Janeto, B. Shereen,et al., "Saturated models of pupildom: assessment and inclusion/exclusion,” British Educational Research Journal, vol.30, no.6,pp.801-817,2004.

[22]P.A. Rebecca, S.H. Vivien. "Academic expectations stress inventory: development, factor analysis, reliability, and validity," Educational and Psychological Measurement,vol.66, no.3,pp.522-539,2006.

[23]R.C. Donna, B.B. Brown. "The multidimensionality of peer pressure in adolescence," Journal of Youth and Adolescence, vol.14, no.6,pp.451-468,1985.

[24]C.B. Paul,P.F. John. "Measuring school-related stressors in adolescents," Journal of Youth and Adolescence,vol.26, no.4,pp.415-428,1997.

[25]J.D. Sun,P.D. Michael, X.Y. Hou, et al. "Educational stress among chinese adolescents: individual, family, school and peer influences," Educational Review, vol.65, no.3,pp.284-302,2013.

[26] L.S.Robert, B.W.W. Janet, G. Miriam,etal., "The structured clinical interview for dsm-iii-r (scid): i: history, rationale, and description," Arch Gen Psychiatry,vol.49, no.8,pp.624-629,1992.

[27]M.A. Martin, J.B. Peter, J.C. Brian, et al.,"Psychometric properties of the 42-item and 21-item versions of the depression anxiety stress scales in clinical groups and a community sample," Psychological Assessment, vol.10, no.2,pp.176-181,1998.

[28]T.B. Aaron, E. Norman, B. Gary,etal.,"An inventory for measuring clinical anxiety: psychometric properties," Journal of Consulting and Clinical Psychology, vol.56, no.6,pp.893-897,1988.

[29]S.M. John, D.A.P. James, S.P.S. Kevin, et al.,"The multidimensional anxiety scale for children(masc): factor structure, reliability, and validity," Journal of the American Academy of Child \& Adolescent Psychiatry,vol.36, no.4,pp.554-565,1997.

[30]N. Yoram. "Determinants and consequences of students' burnout in universities," Journal of HigherEducation, vol.61, no.1,pp.20-31,1990.

[31]M.Christina. "The measurement of experienced burnout," Journal of Occupational Behaviour, no.2,pp.99-113,1981.

ISSN: 0010-8189

(C) CONVERTER 2021

www.converter-magazine.info 
[32]H.Qiao, B.S. Wilmar. "The factorial validity of the maslach burnout inventory-student survey in china," Psychologist, vol.105, no.2,pp.394-408,2009.

[33]B.B. Arnold, D. Evangelia,V. Willem. "Using the Job Demands-resources Model to Predict Burnout and Performance. Human Resource Management, vol.43, no.1,pp.83-104,2004.

[34]M.R. Richard, P.C. James. "Perceived locus of causality and internalization: examining reasons for acting in two domains," Journal of Personality and Social Psychology, vol.57, no.5,pp.749-761,1989.

[35]A.K. William. "Psychological conditions of personal engagement and disengagement at work," Academy of Management Journal, vol.33, no.4,pp.692-724,1990.

[36] J.W. Andrew, G.D. Ronald. "Construct dimensionality of engagement and its relation with satisfaction," The Journal of Psychology, vol.143, no.1,pp. 91-111,2009.

[37]R.B. Brant, L.M Erinaac. "Supportive communication," in Mark L. Knapp, John A.Daly (Eds.)," Handbook of Interpersonal Communication (3rd ed.).Thousand Oaks, CA: Sage, 2002,pp.374-424.

[38]Erina L. MacGeorge, Wendy Samter, Seth J. Gillihan. Brief reportacademic stress, supportive communication, and health. Communication Education, vol.54, no.4,pp.365-372,2005.

[39]C.Sheldon, U.G.Lynn, H.G. Benjamin. "Social support measurement and intervention. Oxford, UK, Oxford University Press, 2000,pp.3-25.

[40]G. Jai, L,Jim, S.Gangaram. "Burnout and personality:evidence from academia," Journal of Career Assessment, vol.15, no.2,pp.240-256,2007.

[41] R. Michael. "A schedule for assessing self-control behaviours: preliminary findings," Behaviour Therapy,vol.11, no.1,pp.109-121,1980.

[42]C.L. Janina. "Coping with job stress: measures and future directions for scale development," Journal of Applied Psychology, vol.71, no.3,pp.377-385,1986.

[43]Donald Meichenbaum. "Stress inoculation training for coping with stressors," The Clinical Psychologist, no.49,pp.4-7,1996.

[44]S.S.Shu. "An investigation into academic burnout among taiwanese adolescents from the self-determination theory perspective," Social Psychology of Education, vol.18, no.1,pp.201-219,2015.

[45]S.Marisa, S.Wilmar, M.Isabel. "How obstacles and facilitators predict academic performance: the mediating role of study burnout and engagement,"Anxiety, Stress \& Coping: An International Journal, vol.23, no.1,pp.53-70,2010. 